\title{
SCIENTIFIC REPORTS

\section{OPEN Antimicrobial Photoinactivation Using Visible Light Plus Water- Filtered Infrared-A (VIS + wIRA) and Hypericum Perforatum Modifies In Situ Oral Biofilms}

\author{
Andreas Vollmer ${ }^{1}$, Ali Al-Ahmad ${ }^{1}$, Aikaterini Argyropoulou ${ }^{2}$, Thomas Thurnheer $\mathbb{B}^{3}$, \\ Elmar Hellwig ${ }^{1}$, Thomas Attin ${ }^{3}$, Kirstin Vach ${ }^{4}$, Annette Wittmer ${ }^{5}$, Kerry Ferguson ${ }^{6}$, \\ Alexios Leandros Skaltsounis ${ }^{2}$ \& Lamprini Karygianni $\mathbb{B}^{3 *}$
}

Due to increasing antibiotic resistance, the application of antimicrobial photodynamic therapy (aPDT) is gaining increasing popularity in dentistry. The aim of this study was to investigate the antimicrobial effects of aPDT using visible light (VIS) and water-filtered infrared-A (wIRA) in combination with a Hypericum perforatum extract on in situ oral biofilms. The chemical composition of $H$. perforatum extract was analyzed using ultra-high-performance liquid chromatography coupled with high resolution mass spectrometry (UPLC-HRMS). To obtain initial and mature oral biofilms in situ, intraoral devices with fixed bovine enamel slabs (BES) were carried by six healthy volunteers for two hours and three days, respectively. The ex situ exposure of biofilms to VIS + wIRA $\left(200 \mathrm{mWcm}^{-2}\right)$ and $H$. perforatum (32 $\mathrm{m} \mathrm{m} \mathrm{ml}^{-1}$, non-rinsed or rinsed prior to aPDT after 2-min preincubation) lasted for five minutes. Biofilm treatment with $0.2 \%$ chlorhexidine gluconate solution (CHX) served as a positive control, while untreated biofilms served as a negative control. The colony-forming units (CFU) of the aPDTtreated biofilms were quantified, and the surviving microorganisms were identified using MALDI-TOF biochemical tests as well as $16 \mathrm{~S}$ rDNA-sequencing. We could show that the $H$. perforatum extract had significant photoactivation potential at a concentration of $32 \mathrm{mg} \mathrm{ml}^{-1}$. When aPDT was carried out in the presence of $H$. perforatum, all biofilms $(100 \%)$ were completely eradicated $(p=0.0001)$. When $H$. perforatum was rinsed off prior to aPDT, more than $92 \%$ of the initial viable bacterial count and $13 \%$ of the mature oral biofilm were killed. Overall, the microbial composition in initial and mature biofilms was substantially altered after aPDT, inducing a shift in the synthesis of the microbial community. In conclusion, $H$. perforatum-mediated aPDT using VIS + wIRA interferes with oral biofilms, resulting in their elimination or the substantial alteration of microbial diversity and richness. The present results support the evaluation of $H$. perforatum-mediated aPDT for the adjunctive treatment of biofilmassociated oral diseases.

The growing ineffectiveness of several conventional antimicrobial agents for the treatment of various biofilm-associated infections has been identified ${ }^{1,2}$. Interestingly, it has been shown that biofilm inhabitants have an enhanced resistance of up to 1,000 times against antibiotics compared with their planktonic counterparts ${ }^{3,4}$. Recently, aPDT was successfully applied to treat biofilm-associated oral infections such as caries, periodontitis

\footnotetext{
${ }^{1}$ Department of Operative Dentistry and Periodontology, Center for Dental Medicine, Albert-Ludwigs-University, Freiburg, Germany. ${ }^{2}$ Department of Pharmacognosy and Chemistry of Natural Products, Faculty of Pharmacy, National and Kapodistrian University of Athens, Athens, Greece. ${ }^{3}$ Clinic for Conservative and Preventive Dentistry, Center of Dental Medicine, University of Zurich, Zurich, Switzerland. ${ }^{4}$ Institute for Medical Biometry and Statistics, Center for Medical Biometry and Medical Informatics, Albert-Ludwigs-University, Freiburg, Germany. ${ }^{5}$ Institute of Medical Microbiology and Hygiene, Albert-Ludwigs-University, Freiburg, Germany. ${ }^{6}$ Botanical Innovation, Unit 2, 390 Clergate Road, Orange, NSW, 2800, Australia. *email: lamprini.karygianni@zzm.uzh.ch
} 


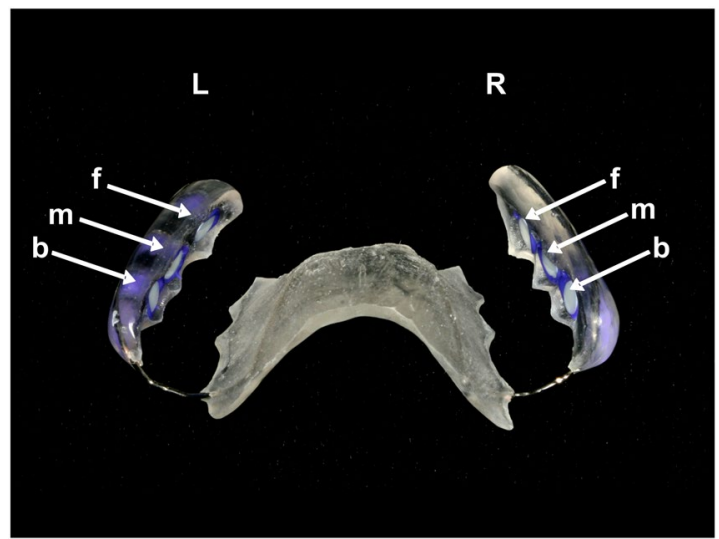

Figure 1. Individual upper jaw acrylic splint. The BES were attached at the front (f), in the middle (m) and at the back (b) on the right (R) and left (L) sides of the splint. Both BES downward-facing surface margins were covered with silicon, while only their upward-facing surfaces remained exposed to the buccal tooth surfaces during the experiments.

and periimplantitis ${ }^{5}$. Thus, aPDT-driven oral decontamination can serve as an adjunct method to traditional dental treatment approaches.

The majority of aPDT protocols involve the combined application of various harmless light sources and mostly cationic local photosensitizing agents such as methylene blue (MB), toluidine blue $\mathrm{O}(\mathrm{TB})$, indocyanine green (ICG) and chlorine e6 (Ce6) ${ }^{6-8}$. Regarding the light sources, the combination of visible light (VIS) and water-filtered infrared-A (wIRA) has been shown to increase in situ temperature and perfusion levels, inducing wound healing at higher oxygen partial tissue pressure. Interestingly, VIS + wIRA correlates with significant pain reduction ${ }^{9}$. Another advantage of wIRA is the induction of low thermal stress via sufficient tissue penetration, which protects the external tissue layers ${ }^{10,11}$. Already-tested photosensitizers such as TB and Ce6 have shown enhanced antimicrobial activity in combination with aPDT against monospecies and multispecies biofilms ${ }^{12-15}$. Recently, VIS + wIRA in combination with TB or Ce6 has proven to be a potent alternative treatment against in situ supragingival and subgingival multispecies biofilms ${ }^{16,17}$.

The photodynamic properties of Hypericum perforatum (St. John's Wort) have been well known for years now $^{18,19}$. Indeed, it is one of the most commonly-investigated medicinal plants of the last two decades ${ }^{20-22}$. To date, $H$. perforatum has been widely used for topical application to skin prior to aPDT to accelerate chronic wound healing ${ }^{18}$. H. perforatum can also be used as an antidepressive substance ${ }^{23,24}$. Due to its additional favorable anti-oxidant, anti-inflammatory, anti-cancer and antimicrobial properties, $H$. perforatum is responsible for the enhanced differentiation of keratinocytes ${ }^{18}$. Interestingly, its high biocompatibility could lead it to play an outstanding role in aPDT application in the dental field ${ }^{25}$. Among $H$. perforatum's main components, lipophilic hyperforin and hypericin seem to be the most active substances ${ }^{26,27}$. Favorably, H. perforatum shows no mutagenic potential and binding to DNA molecules ${ }^{28}$.

Despite their excellent properties, common photosensitizers such as TB or Ce6 fail to efficiently penetrate into extracellular DNA within the polysaccharide matrix to eradicate the pathogens situated in the deepest biofilm layers ${ }^{29}$. In this context, the photodynamic properties of $H$. perforatum prompted the idea that aPDT using VIS + wIRA and $H$. perforatum could be a promising alternative method for treating multispecies oral biofilms. Considering that the development of oral diseases such as periodontitis and periimplantitis relates to pathological shifts in oral biofilms, modifying biofilm composition and thus diversity could positively affect the treatment impact $^{23}$.

The aim of the present study was to investigate the survival and diversity of microorganisms within initial and mature oral biofilms after the application of aPDT using VIS + wIRA and H. perforatum as a photosensitizer. For this approach, we cultivated oral biofilms in situ onto bovine enamel samples (Fig. 1) for two hours (h) and three days (d), respectively. The in situ biofilms were then treated with the photosensitizer $H$. perforatum and VIS + wIRA ex vivo aPDT (Fig. 2). To our knowledge, alteration in biofilm diversity following aPDT by VIS + wIRA and $H$. perforatum has not been studied to date. The null hypothesis was that aPDT using VIS + wIRA and $H$. perforatum would not eliminate oral biofilms or modify the composition of in situ oral biofilms.

\section{Results}

Chemical composition of the $\boldsymbol{H}$. perforatum extract. A rapid and accurate method was developed, aiming to detect the major active compounds in the $H$. perforatum extract, as described elsewhere ${ }^{30}$. For the LC-HRMS, an Orbitrap high resolution mass analyzer was used in negative ionization mode. Peaks were identified by comparing the retention times and HRMS data of the compounds with already-published data ${ }^{31,32}$ and natural product databases (PubChem, ChemSpider). The extract of $H$. perforatum contains a wide range of constituents (Table 1, Fig. 3). All of the detected compounds along with their names, Rt (min), molecular formula, theoretical and experimental $\mathrm{m} / \mathrm{z}$, mass difference (ppm) and RDBeq (Relative Double Bond equivalent) value are summarized in Table 1 , whereby a total of sixteen secondary compounds were detected. Phenolic 


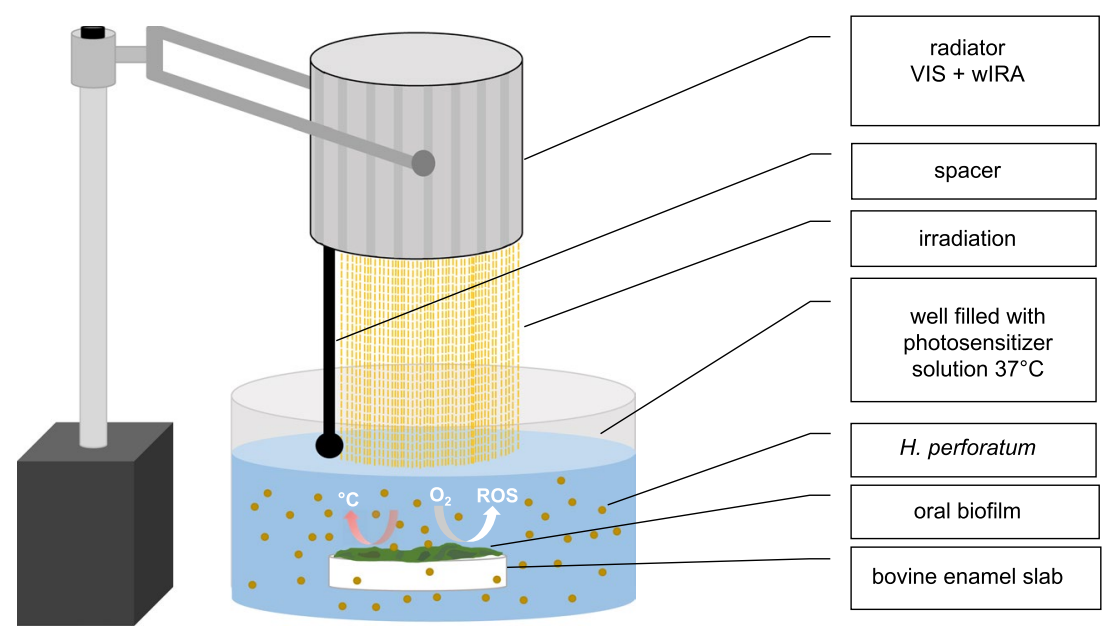

Figure 2. Graphical scheme depicting aPDT using VIS + wIRA as a light source and $H$. perforatum as a photosensitizer against oral biofilms. The VIS + wIRA with a broadband water-filtered spectrum $(570-1400 \mathrm{~nm})$ facilitated the excitation of the photosensitizing agent $(H$. perforatum), triggering its interaction with oxygen $\left(\mathrm{O}_{2}\right)$. ROS were then released, eradicating both planktonic and adherent oral microorganisms.

\begin{tabular}{|c|c|c|c|c|c|c|c|}
\hline \multirow[b]{2}{*}{ No } & \multirow[b]{2}{*}{ Rt (min) } & \multirow[b]{2}{*}{$\mathbf{E C}[\mathbf{M}-\mathbf{H}]^{-}$} & Experimental & Theoretical & \multirow[b]{2}{*}{$\Delta \mathrm{m} \mathrm{ppm}$} & \multirow[b]{2}{*}{ RDBeq } & \multirow[b]{2}{*}{ Identification of compound } \\
\hline & & & \multicolumn{2}{|l|}{$[\mathrm{M}-\mathrm{H}]^{-} m / z$} & & & \\
\hline 1 & 0.65 & $\mathrm{C}_{7} \mathrm{H}_{11} \mathrm{O}_{6}$ & 191.0544 & 191.0561 & -8.730 & 2.5 & Quinic acid \\
\hline 2 & 6.14 & $\mathrm{C}_{16} \mathrm{H}_{17} \mathrm{O}_{9}$ & 353.0835 & 353.0819 & -12.363 & 8.5 & Chlorogenic acid \\
\hline 3 & 7.60 & $\mathrm{C}_{17} \mathrm{H}_{19} \mathrm{O}_{9}$ & 367.0990 & 367.0976 & -12.137 & 8.5 & 3-O-Feruloylquinic acid \\
\hline 4 & 8.08 & $\mathrm{C}_{21} \mathrm{H}_{19} \mathrm{O}_{12}$ & 463.0826 & 463.0823 & 0.615 & 12.5 & Hyperoside \\
\hline 5 & 8.16 & $\mathrm{C}_{21} \mathrm{H}_{19} \mathrm{O}_{12}$ & 463.0828 & 463.0823 & 1.077 & 12.5 & Isoquercitrin \\
\hline 6 & 8.67 & $\mathrm{C}_{21} \mathrm{H}_{19} \mathrm{O}_{11}$ & 447.0880 & 447.0874 & -12.536 & 12.5 & Isoquercetrin \\
\hline 7 & 8.72 & $\mathrm{C}_{21} \mathrm{H}_{19} \mathrm{O}_{11}$ & 447.0877 & 447.0874 & -12.334 & 12.5 & Quercitrin \\
\hline 8 & 10.09 & $\mathrm{C}_{15} \mathrm{H}_{9} \mathrm{O}_{7}$ & 301.0319 & 301.0295 & -11.480 & 11.5 & Quercetin \\
\hline 9 & 10.36 & $\mathrm{C}_{27} \mathrm{H}_{29} \mathrm{O}_{16}$ & 609.1388 & 609.1402 & -1.965 & 13.5 & Rutin \\
\hline 10 & 11.41 & $\mathrm{C}_{30} \mathrm{H}_{17} \mathrm{O}_{10}$ & 537.0762 & 537.0827 & -12.195 & 22.5 & I3,II8 Biapigenin \\
\hline 11 & 12.04 & $\mathrm{C}_{30} \mathrm{H}_{17} \mathrm{O}_{10}$ & 537.0757 & 537.0827 & -12.994 & 22.5 & Amentoflavone \\
\hline 12 & 14.34 & $\mathrm{C}_{36} \mathrm{H}_{27} \mathrm{O}_{15}$ & 699.1268 & 699.1355 & -12.463 & 23.5 & Skyrin-2-O-glucopyranoside \\
\hline 13 & 20.37 & $\mathrm{C}_{30} \mathrm{H}_{43} \mathrm{O}_{4}$ & 467.3113 & 467.3167 & -11.564 & 9.5 & Hyperfirin \\
\hline 14 & 22.20 & $\mathrm{C}_{35} \mathrm{H}_{51} \mathrm{O}_{4}$ & 535.3730 & 535.3793 & -11.649 & 10.5 & Hyperforin \\
\hline 15 & 22.74 & $\mathrm{C}_{30} \mathrm{H}_{15} \mathrm{O}_{8}$ & 503.0715 & 503.0714 & -11.352 & 23.5 & Hypericin \\
\hline 16 & 23.27 & $\mathrm{C}_{30} \mathrm{H}_{15} \mathrm{O}_{9}$ & 519.0661 & 519.0663 & -11.723 & 23.5 & Pseudohypericin \\
\hline
\end{tabular}

Table 1. Chromatographic and spectrometric characteristics of secondary metabolites identified in $H$. perforatum by LC(ESI-)HRMS.

acids, flavonoids, biflavones, phloroglucinols and naphthodianthrones were identified. Naphthodianthrones are characteristic compounds of the genus Hypericum. In the studied extract, hypericin and pseudohypericin were detected at Rt 22.74 and $23.27 \mathrm{~min}$, respectively. Phloroglucinol - represented by hyperfirin and hyperforin was also identified, as they are considered typical representatives of the genus. Several types of flavonoids - a flavonol (quercetin), flavonoid-O-glycosides (isoquercetrin, quercitrin, rutin and skyrin-2-O-glucopyranoside) and flavonol galactosides (hyperoside and isoquercitrin) - were identified. Two dimeric types of flavones - I3,II8 Biapigenin at Rt $11.41 \mathrm{~min}$ and amentoflavone at Rt $12.04 \mathrm{~min}$ - that are commonly detected in $H$. perforatum were also found in the studied extract. Additionally, various phenolic compounds (quinic acid, chlorogenic acid and 3-O-feruloylquinic acid) were detected.

aPDT effects on the viable counts of oral microorganisms at initial adhesion and in mature oral biofilms. Figure 4a shows the effects of aPDT using VIS + wIRA and H. perforatum on the log counts of the initial and mature oral biofilms after two hours and three days, respectively. The aPDT induced a complete eradication of the bacteria ( $\mathrm{p}=0.0001$, Fig. $4 \mathrm{a}$ ) within the initial biofilms treated with non-rinsed H. perforatum, whether followed or not by aPDT, as well as within the CHX-treated initial biofilms. Interestingly, the initial biofilms in which $H$. perforatum was rinsed prior to aPDT exhibited a mean of $0.69 \pm 0.95 \mathrm{CFU}$ in the $\log _{10}$ scale 

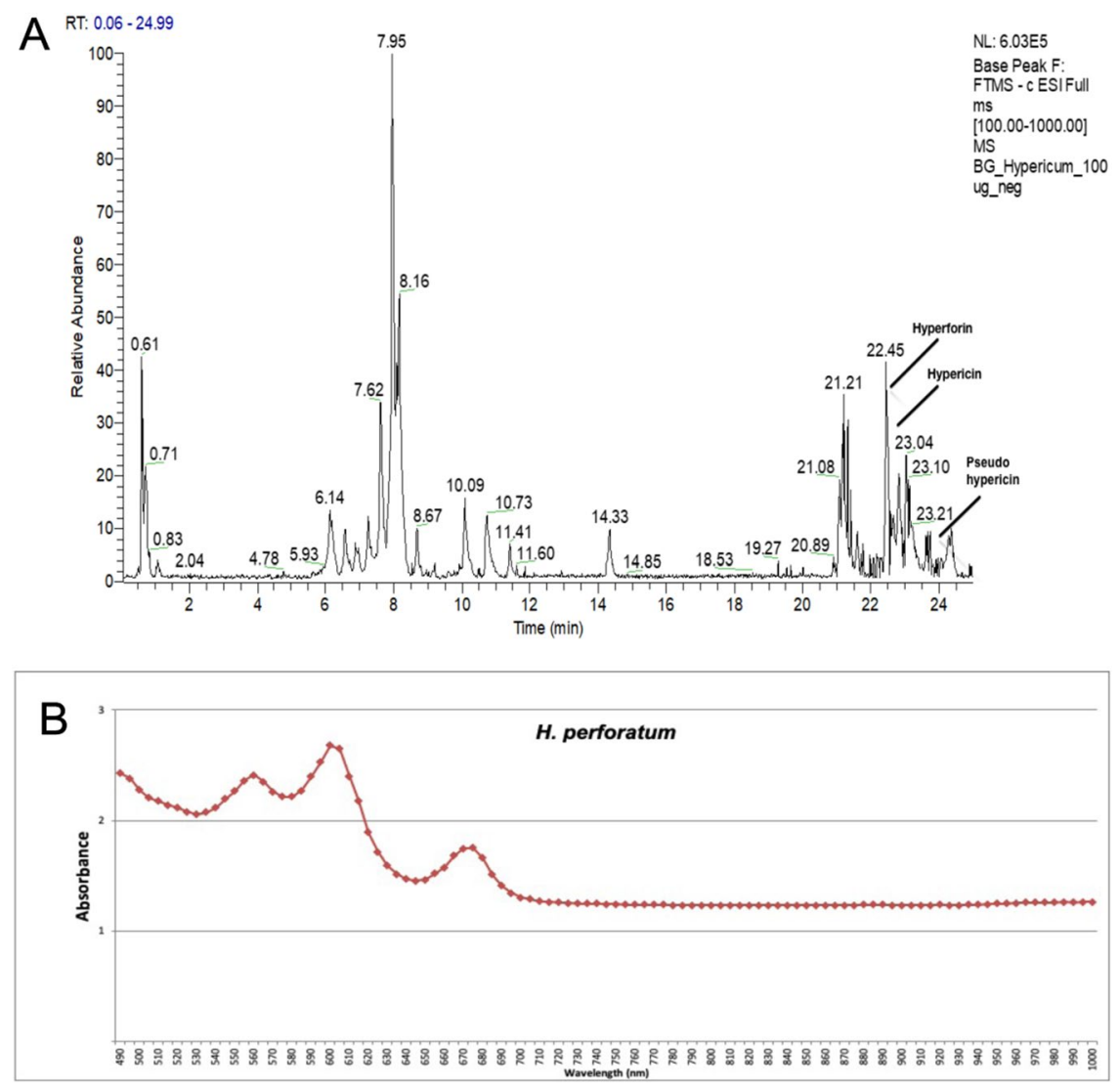

Figure 3. Panel A depicts UPLC-ESI-HRMS chromatogram of the $H$. perforatum extract. The peaks of hyperforin, hypericin and pseudohypericin are marked on the graph. Panel B depicts the absorbance spectrum of the fabricated $H$. perforatum extract including $16 \%$ alcohol. The concentration of $H$. perforatum was $32 \mathrm{mg} \mathrm{ml}^{-1}$. The measurement of the spectrum was conducted using a Tecan Infinite 200 reader (Tecan, Crailsheim, Germany).

(median, 0.25), which is a significantly reduced $(\mathrm{p}=0.002)$ microbial growth compared with the untreated negative control (mean, $3.81 \pm 0.66$; median, 3.75).

Figure 4(b,c) displays the microbial growth rates of the three-day-old oral biofilm after the application of aPDT. In the presence of $H$. perforatum, the aerobic and anaerobic microorganisms within the aPDT-treated biofilms showed no microbial growth and thus a CFU reduction of $100 \%(\mathrm{p}=0.0001)$. With regard to the aerobic biofilm bacteria, aPDT following rinsing of $H$. perforatum (mean, $5.49 \pm 0.55$; median, 5.52) yielded no significant CFU decline compared with the untreated negative control (mean, $6.23 \pm 0.38$; median, 6.17) and the CHX-treated positive control (mean, $5.44 \pm 0.71$; median, 5.45). Regarding the anaerobic biofilm bacteria, the application of aPDT after rinsing $H$. perforatum (mean, $4.49 \pm 1.00$; median, 4.64 ) failed to yield a decrease in the $\log _{10}$ CFU counts compared with the negative control (mean, 4.49 \pm 1.06 ; median, 4.32), although it proved to be as effective as CHX (mean, 3.19 \pm 2.17 ; median, 4.04). The application of $H$. perforatum only without subsequent aPDT had no significant effect on either aerobic (mean, 5.02 \pm 1.18 ; median, 5.39) or anaerobic (mean, $5.33 \pm 2.17$; median, 4.04) microorganisms.

Shift of bacterial spectrum after the use of aPDT against initial microbial adhesion. The composition of cultivable bacteria in initial adhesion samples is analyzed in Figs. 5A,B, 6A, and 7A,B. After treatment with CHX (positive control) and $H$. perforatum, whether followed or not by aPDT, all bacteria were eliminated. From the original bacterial community in the untreated negative control, a total of 31 different cultivable species were isolated and identified, as shown in Fig. 5A. The microbial community was predominated (29\%) by eight different streptococcal species (Streptococcus mitis/oralis 11\%; Streptococcus sanguinis/parasanguinis, 7\%; Streptococcus salivarius, 4\%; Streptococcus australis, 3\%; Streptococcus infantis, 3\%; Streptococcus gordonii, 1\%). Different species of the genera Rothia (Rothia dentocariosa, Rothia aeria, Rothia mucilaginosa), Actinomyces (Actinomyces odontolyticus, Actinomyces graevenitzii, Actinomyces oris, Actinomyces naeslundii), Neisseria (Neisseria flavescens/perflava, Neisseria mucosa, Neisseria macacae) and Gemella (Gemella sanguinis, Gemella haemolysans) were found in $22 \%, 14 \%, 10 \%$ and $5 \%$ of the isolated bacteria, respectively. The percentages of all other recovered species mainly belonging to the genera Veillonella, Capnocytophaga and Campylobacter, ranged from $2-8 \%$. As depicted in Fig. 5B, the aPDT with VIS + wIRA and H. perforatum - which was rinsed prior to aPDT - reduced the number of viable bacterial species from 31 to 8, comprising S. sanguinis/parasanguinis (31\%), S. mitis/oralis (23\%), R. mucilaginosa (22\%), N. mucosa/macacae (12\%) and Granulicatella elegans (12\%). 


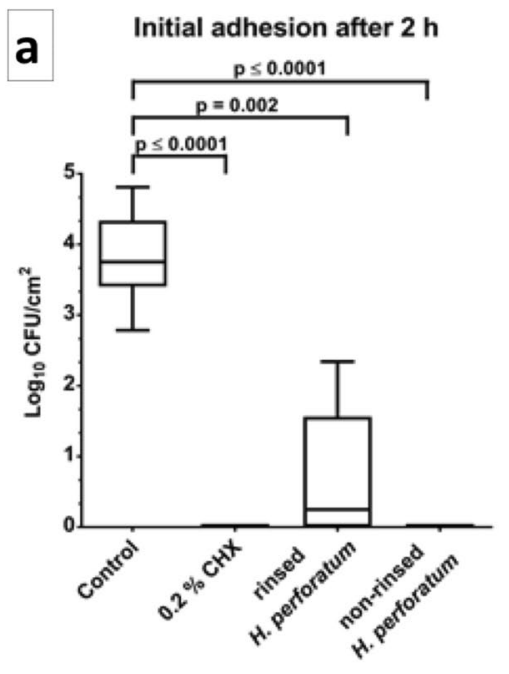

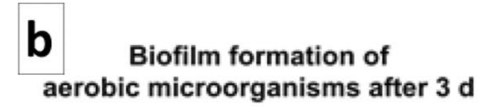

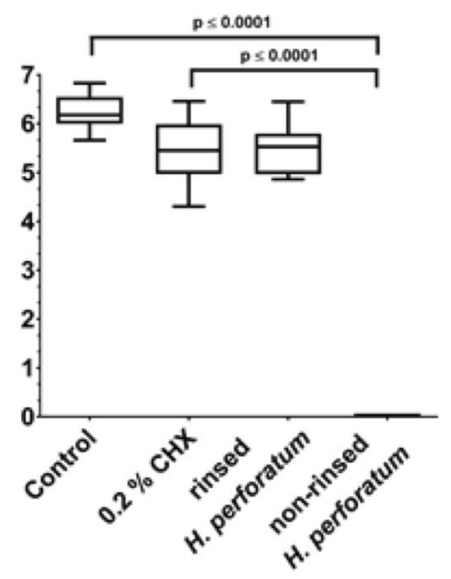

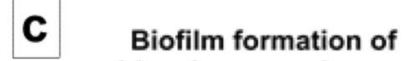
anaerobic microorganisms after $3 \mathrm{~d}$

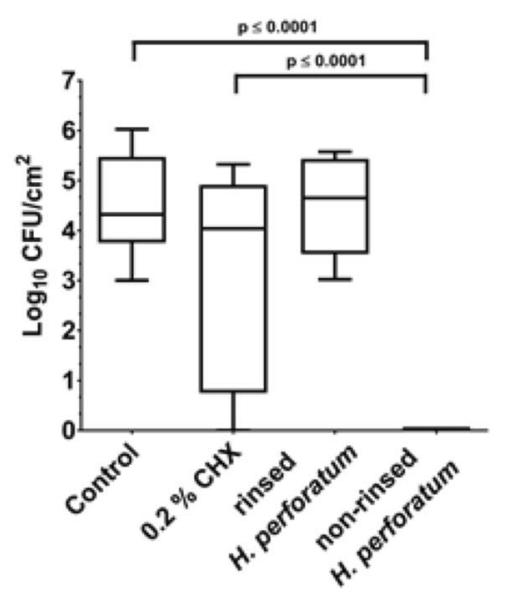

Figure 4. Boxplots of CFU counts, demonstrating the photodynamic efficiency (aPDT using VIS + wIRA and $H$. perforatum) against oral microorganisms after two-hour $(2 \mathrm{~h})$ initial adhesion (a) as well as against aerobic (b) and anaerobic (c) microorganisms following three-day ( $3 \mathrm{~d}$ ) biofilm formation. The bacterial counts of the following treatment groups are demonstrated for two-hour- and three-day-old oral biofilms: untreated and $H$. perforatum-treated negative controls, $0.2 \%$ CHX-treated positive control, aPDT after rinsing off $H$. perforatum, and aPDT without rinsing off $H$. perforatum. The CFUs are presented on a $\log _{10}$ scale per square centimeter $\left(\log _{10} / \mathrm{cm}^{2}\right)$. The p-values ( $\mathrm{t}$ test) of the significantly different data are marked on the graphs.
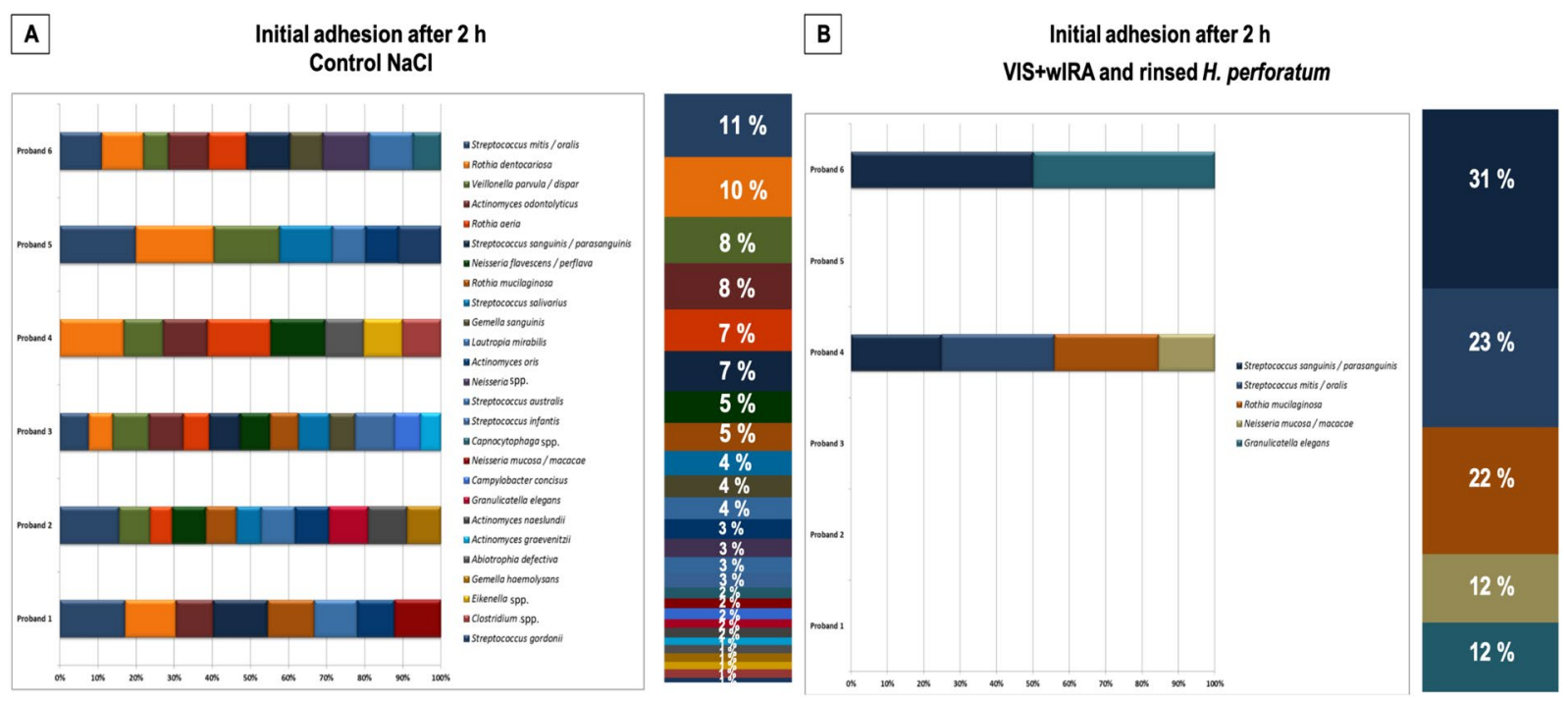

Figure 5. Relative distribution among the volunteers and total distribution (in percentages) of bacterial species among six probands after two-hour $(2 \mathrm{~h})$ initial adhesion following aPDT using VIS + wIRA and $H$. perforatum. Graph A depicts the bacterial composition of the untreated negative controls after initial adhesion. Graph B shows the bacterial diversity after aPDT against initially adhered bacteria. Consistent color coding was used for the study participants and bacterial species, as indicated by the schemes at the right of panels, respectively.

In the presence of reduced microbial richness, the species diversity of the surviving bacteria among the probands was also strongly affected. The most prevalent species in the untreated control group were Streptococcus mitis/oralis, $R$. dentocariosa and Veillonella parvula/dispar (detected in five volunteers), followed by A. odontolyticus and R. aeria (retrieved from four volunteers). All other species were detected in three volunteers or fewer. After pre-treatment with $H$. perforatum, rinsing and subsequent aPDT with VIS + wIRA, no cultivable bacteria were detected in four of the volunteers. The two remaining positive samples were mainly comprised of $S$. sanguinis/parasanguinis and G. elegans, whereas seven different species were only detectable in the second volunteer. 
A Initial adhesion

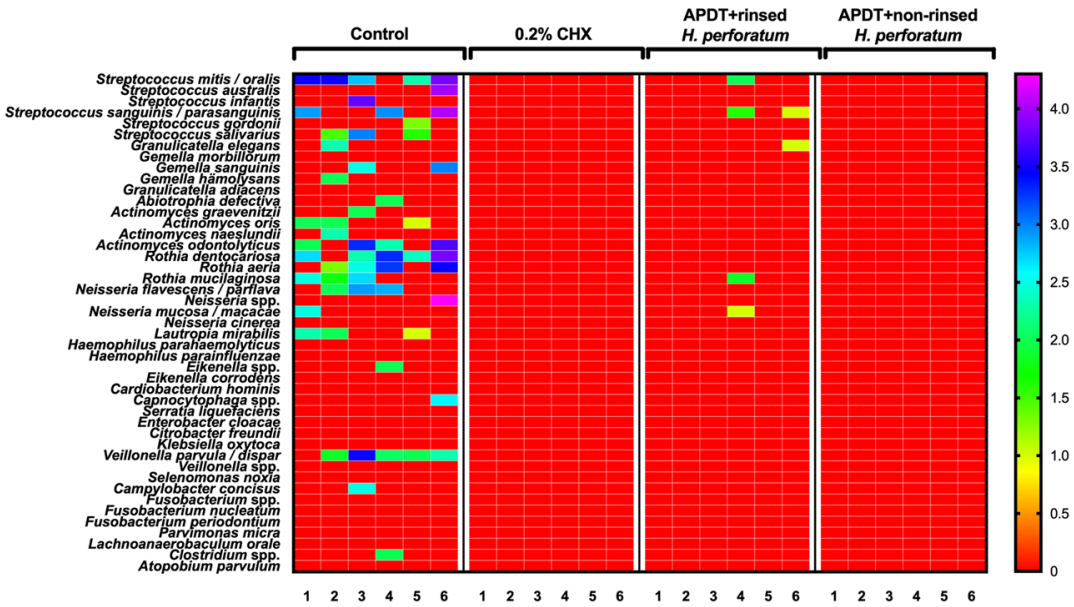

B

\section{Oral biofilm}

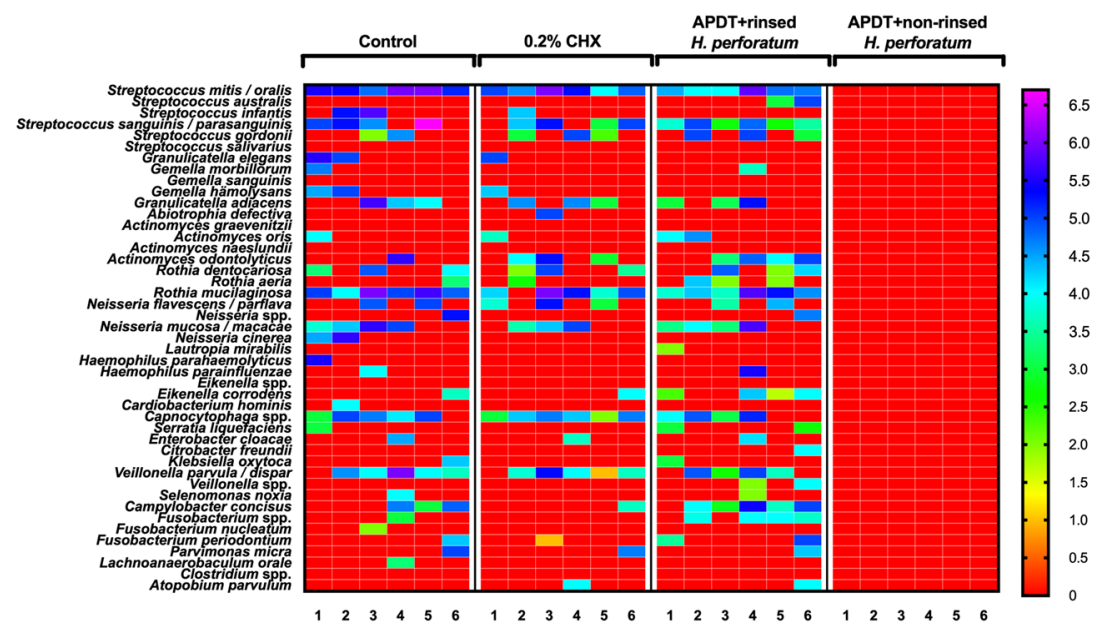

Figure 6. Heatmap presenting the absolute distribution (in $\log _{10} / \mathrm{cm}^{2}$ ) of bacterial species among six study probands after two-hour $(2 \mathrm{~h})$ initial adhesion and three-day $(3 \mathrm{~d})$ biofilm formation following aPDT using VIS + wIRA and $H$. perforatum. The photodynamic efficiency against oral microorganisms after initial adhesion and three-day biofilm formation is demonstrated in panels $\mathrm{A}$ and $\mathrm{B}$, respectively. The bacterial composition of the following treatment groups is demonstrated for two-hour- and three-day old oral biofilms: untreated negative control, $0.2 \% \mathrm{CHX}$-treated positive control, aPDT after rinsing off $H$. perforatum, aPDT without rinsing off $H$. perforatum. Proband numbers for each treatment group $(n=6)$ are shown in columns and variables (bacterial species and genera) are shown in rows. The variations in the colors depicted on the color scale bars on the right indicate how data values change from very low to extremely high.

Shift of bacterial community composition after aPDT application to the mature oral biofilm. Interestingly, after aPDT with VIS + wIRA in the presence of H. perforatum, the total microbial load of the biofilms was reduced by $100 \%$. The microbial richness after pre-treatment with $H$. perforatum and aPDT with VIS + wIRA and the bacterial composition of the controls (negative: untreated, positive: treatment with $0.2 \%$ $\mathrm{CHX}$ ) are illustrated in Figs. 8A-C, 6B and 7C-E. Regarding the microbial richness within the mature biofilms, the untreated control, the CHX-treated positive control and the aPDT-treated biofilms comprised 35, 27, and 30 different bacterial species, respectively.

The microbial community in the negative control group (Fig. 8A) was predominated (24\%) by six different streptococcal species (S. mitis/oralis, 11\%; S. sanguinis/parasanguinis, 7\%; S. infantis, 4\%; S. gordonii, 2\%). Different species of the genera Rothia ( $R$. dentocariosa, $R$. aeria, $R$. mucilaginosa), Neisseria (N. flavescens/perflava, N. mucosa/macacae, N. cinerea), Veillonella (V.parvula/dispar) and Granulicatella (G. adiacens, G. elegans) were found in $15 \%, 14 \%, 8 \%$ and $8 \%$ of the isolated bacteria, respectively. The percentages of all other recovered species mainly belonging to the genera Capnocytophaga, Campylobacter, Gemella (G. sanguinis, G. haemolysans) and Actinomyces (A. odontolyticus, A. oris) ranged from 3 to $7 \%$.

In the positive control (Fig. 8B), the microbial richness was reduced to 27 species after treatment with $0.2 \%$ CHX. Although the counts of Eikenella corrodens, S. gordonii, and R. dentocariosa increased after the CHX 


\section{2-hour initial adhesion}

A

B

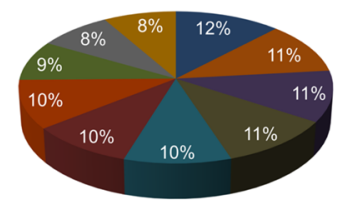

$\mathrm{NaCl}$

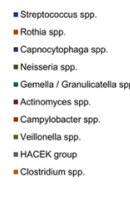

3-day oral biofilm
C

$\mathrm{NaCl}$

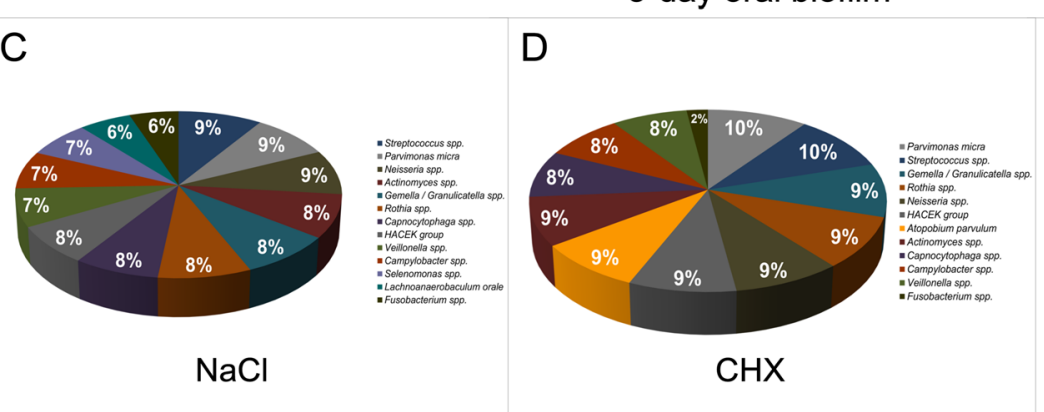

D

$\mathrm{CHX}$

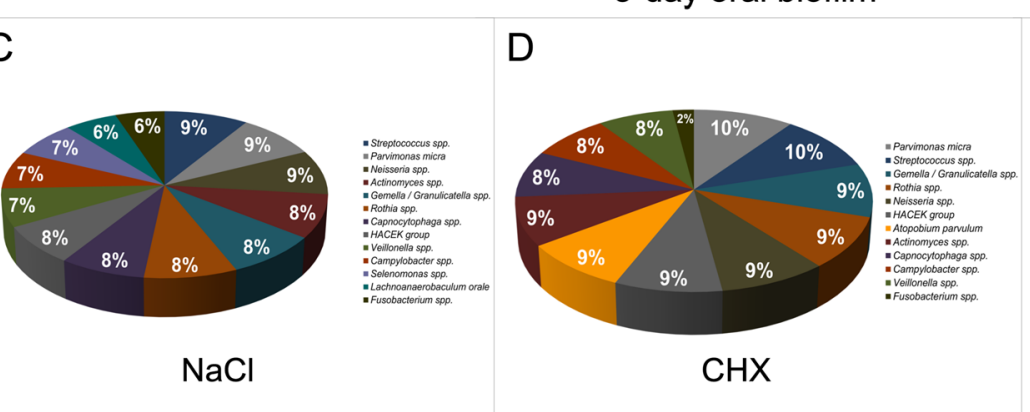

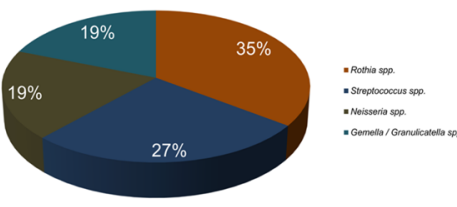

HP rinsed

$E$

Figure 7. Pie charts demonstrating the total distribution (in percentages) of bacterial genera among six probands after two-hour $(2 \mathrm{~h})$ initial adhesion and three-day $(3 \mathrm{~d})$ biofilm formation following aPDT using VIS + wIRA and $H$. perforatum. Graph A depicts the bacterial composition of the untreated negative controls after initial adhesion. Graph B shows the bacterial diversity after aPDT against initial biofilms. Graphs C-E show the bacterial composition of three-day old biofilms divided into the following groups: the untreated negative control (C), after treatment with $0.2 \%$ CHX (D), and after aPDT (E) with VIS + wIRA and $H$. perforatum (was rinsed off prior to aPDT). Consistent color coding was used for the bacterial genera, as indicated by the schemes at the right of panels A-E, respectively.

treatment, all of the other bacterial counts within the positive control decreased. A total of ten species could not be detected after treatment with CHX, whereas Abiotropha defectiva and Atopobium parvulum could only be detected after the treatment with CHX.

As depicted in Fig. 8C, the aPDT with VIS + wIRA and H. perforatum - which was rinsed prior to aPDT - reduced the number of viable bacterial species from 35 to 30. The aPDT-treated biofilm mainly comprised 17\% Streptococcus spp. (S. mitis/oralis, 5\%; S. sanguinis/parasanguinis, 4\%; S. australis, 4\%; S. gordonii, 4\%), 13\% Neisseria spp. (N. flavescens/perflava, 4\%; N. mucosa/macacae, 4\%) and 12\% Rothia spp. (R. mucilaginosa, 5\%; R. dentocariosa, 4\%; R. aeria, 3\%). The percentages of the other detected genera such as Haemophilus, Campylobacter, Gemella, Veillonella, Capnocytophaga and Actinomyces varied from 4 to 5\%. A total of 15 out of the 30 recovered bacterial species were found to decrease in number. Nine species - Haemophilus parahaemolyticus, N. cinerea, Cardiobacterium hominis, Selenomonas noxia, Lachnoanaerobaculum orale, Fusobacterium spp., S. infantis, G. elegans and G. haemolysans - completely disappeared after the aPDT treatment with H. perforatum, whereas S. australis, Veillonella spp., Atopobium parvulum and Lautropia mirabilis only appeared after the photodynamic application of $H$. perforatum.

Regarding the species distribution among the probands, S. mitis/oralis, R. mucillaginosa and V. parvula/dispar were detected in all six volunteers of the untreated control group, with a total distribution ranging from 8 to $11 \%$. Capnocytophaga spp. (7\%) was detected in five volunteers, whereas the other bacterial species were recovered from 1-4 probands. After the aPDT treatment, S. mitis/oralis, S. sanguinis/parasanguinis, R. mucillaginosa, V. parvula/dispar and Campylobacter concisus were detected in all six volunteers, with a total distribution ranging from 4 to $11 \%$. All other species appeared in 1-5 volunteers and ranged from 1 to $4 \%$. After aPDT treatment, a total of eight aerobic and three anaerobic microorganisms increased in their relative distribution compared with the CHX treatment, where only three aerobic microorganisms increased. In terms of the new appearance of bacteria after the aPDT with $H$. perforatum, two aerobic and two anaerobic bacteria emerged compared with the CHX treatment, where only one aerobic and one anaerobic bacteria appeared.

\section{Discussion}

The present study has established an effective method for microbial photoinactivation combining VIS + wIRA with the natural photosensitizing agent $H$. perforatum to treat in situ-formed initial and mature oral biofilms for the first time. The aPDT combining VIS + wIRA and photosensitizing agents such as TB and Ce6 was reported to successfully photoinactivate oral pathogenic microorganisms situated even in the deepest niches of $i n$ situ supragingival and subgingival biofilms in earlier own reports ${ }^{14,15,17,33}$. In an in vitro study investigating the effectiveness of $H$. hypericum-mediated aPDT on E. faecalis culture in planktonic form and biofilm, a significant microbial 

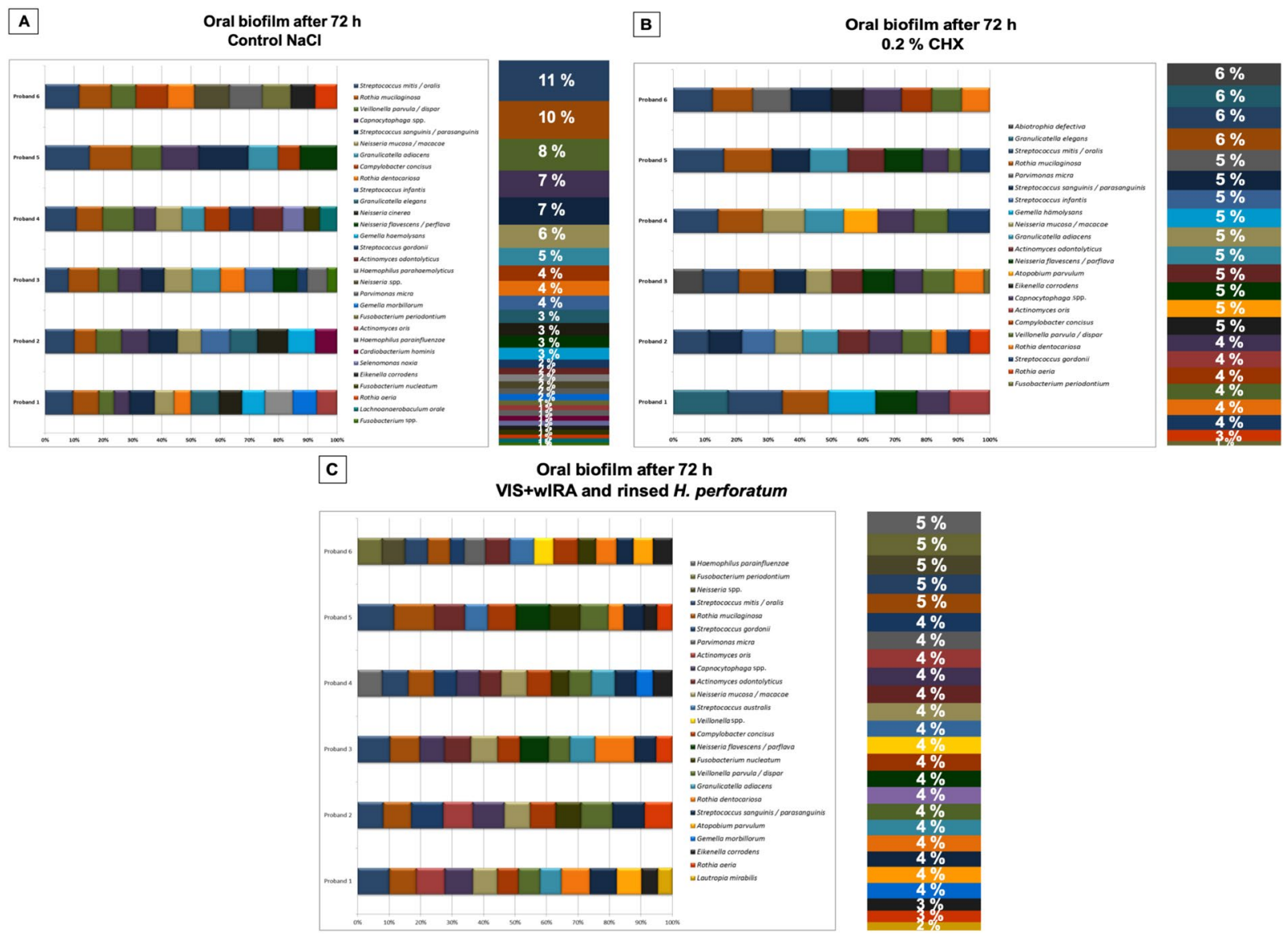

Figure 8. Relative distribution among the volunteers and total distribution (in percentages) of bacterial species among six probands after three-day $(3 \mathrm{~d})$ biofilm formation following aPDT using VIS + wIRA and $H$. perforatum. Graphs A-C demonstrate the bacterial composition of three-day old biofilms divided into the following groups: the untreated negative control (A), after treatment with $0.2 \% \mathrm{CHX}(\mathbf{B})$, and after aPDT (C) with VIS + wIRA and $H$. perforatum (was rinsed off prior to aPDT). Consistent color coding was used for the study participants and bacterial species, as indicated by the schemes at the right of panels A-C, respectively.

reduction of up to $5 \log$ counts was detected ${ }^{34}$. The innovative contribution of this study is that, to the best of our knowledge, $H$. perforatum - widely known for its anti-depressant properties - is here studied for the first time in combination with VIS + wIRA as a light source to treat in situ oral biofilms ${ }^{35-38}$.

For an effective aPDT light source, visible light (VIS) with an accessory orange filter within the range of 570-780 nm was combined with water-filtered infrared-A (wIRA) to accentuate the amount of infrared-A radiation within the range of 780-1400 $\mathrm{nm}^{39}$. Since PS excitation is only possible within the range of VIS, the use of wIRA alone would not have yielded the desired photodynamic outcomes ${ }^{39}$. The advantages of using VIS + wIRA contrary to VIS alone have already been outlined in the literature ${ }^{9}$. The emission of energy in the form of light results in electron transfer reactions between the substrate and the PS, with the latter entering an electronically excited state ${ }^{40}$. The leading antimicrobial effect of aPDT is the subsequent production in elevated rates of reactive oxygen species (ROS) such as superoxide ion $\left(\mathrm{O}_{2}{ }^{-}\right)$, hydrogen peroxide $\left(\mathrm{H}_{2} \mathrm{O}_{2}\right)$ and free hydroxyl radicals $(\cdot \mathrm{HO})$, all yielded via Type I mechanisms ${ }^{41}$. In a Type II reaction, single oxygen radicals $\left({ }^{1} \mathrm{O}_{2}\right)$ are yielded after the reaction of triplet state PS and oxygen $\left(\mathrm{O}_{2}\right)^{42}$.

Due to the short lifetime of ROS (about 10-320 ns), their diffusion after using conventional light sources (laser and non-laser) is limited and thus the direct aPDT-induced damage remains localized to the PS-infiltrated area $^{43}$. VIS + wIRA also allows for the release of the protective protein ferritin ${ }^{44}$ to prevent cell destruction. The high safety of VIS + wIRA radiators can be guaranteed for treatment times of up to $30 \mathrm{~min}^{45}$, although 5 minutes proved to be a potent average aPDT duration in our report with a focus on daily clinical use. To facilitate a better distribution of photosensitizer within the bacterial cells and to achieve clinically meaningful treatment times, the biofilms were pre-exposed to $H$. perforatum for $2 \mathrm{~min}$ in the dark. In a systematic review, Fumes et al. described pre-irradiation times for methylene blue $(\mathrm{MB})$ between 1 and 15 minutes, with optimal outcomes between 5 and $15 \mathrm{~min}^{46}$. However, there is no published data on the pre-irradiation incubation of $H$. perforatum, which exhibits enhanced cell penetration when diluted in ethanol ${ }^{47}$. Finally, VIS + wIRA raises levels of physiological activity of the regulatory enzyme cytochrome c oxidase with absorption maxima at $620,680,760$ and $825 \mathrm{~nm}^{48}$. 
Due to its numerous advantageous properties, $H$. perforatum constitutes a multipurpose plant ${ }^{49}$ and is considered one of the most well-known natural photosensitizers in different medical fields ${ }^{50}$. Interestingly, $H$. perforatum has proven extremely effective in the treatment of non-melanoma skin cancer as well as other dermal and internal cancer species ${ }^{18,51,52}$. Besides its excellent anti-oxidant and anti-inflammatory effects ${ }^{53}, H$. perforatum has been successfully applied in dentistry for the pain management of trigeminal neuralgia ${ }^{54}$. Furthermore, hypericin and hyperforin - the main components of $H$. perforatum - are responsible for enhanced anti-bacterial activity against Gram-positive and Gram-negative microorganisms in both planktonic and biofilm forms ${ }^{55}$. As a result of its beneficial features, $H$. perforatum can be integrated in a greater number of clinical photoinactivation protocols compared with Ce6, TB, ICG and $\mathrm{MB}^{18,35,37,56}$. When administered systematically, $H$. perforatum inevitably interacts with several cytochrome enzymes, inducing lower plasma levels of anti-cancer agents or other medicines ${ }^{57}$. Nevertheless, local application of $H$. perforatum is associated with fewer side effects regarding cytotoxicity and cross-reactivity with other medicament $s^{58}$. Even after the combined oral application of $H$. perforatum using both a single- and double-dose of UV radiation, visible light, and solar-simulated radiation, no significant pathogenic alterations could be detected at erythema threshold levels ${ }^{59}$. However, a recent report highlighted the increase of round fibroblastic cells, which indicated high cytotoxicity in concentrations of $H$. perforatum higher than $10-50 \mathrm{mg} / \mathrm{ml}^{60}$.

Solvent extraction is the most common means of extracting Hypericum from the flowers of $H$. perforatum (St John's Wort). Solvents include methanol, ethanol, ethyl acetate and acetone. Scientific studies indicate when using extraction solvents containing more than $50 \%$ ethanol or methanol in water the content of the flavonoids in particular Hypericum and Hypericin in the extracts is similar independent of the extraction solvent ${ }^{61}$. H. perforatum extracts are often purified by removing the solvent and then dried into powders prior to commercial sale. This project required a crude liquid extract which contained the solvent for further clinical trials. For this reason methanol, ethyl acetate and acetone were not used as the solvent for the extraction of Hypericum due to the potential toxicity of the solvent in the crude extract. In order to extract bioactive constituents from $H$. perforatum, organic solvents were preferred, as ethanolic $H$. perforatum extracts showed the highest anti-bacterial potential against diverse Gram-positive and Gram-negative bacteria ${ }^{37}$. In the literature, other H. perforatum hydroethanolic extracts such as tinctures with a high alcohol content ranging from 45 to $50 \%$ have been widely used in complementary medicine ${ }^{62}$. In a recent report, $60 \%$ or above of ethanol was utilized to obtain the highest yields of lipophilic hyperforin ${ }^{63}$. However, the use of $100 \%$ (absolute) ethanol led to a reduction of specific hydrophilic components such as flavonoids ${ }^{18}$. Since bacteria in biofilms are more resistant to antimicrobials than their planktonic counterparts, we did not expect a significant decrease of biofilm viability after its exposure to $16 \%$ alcohol solution for seven minutes as detected by CFU. To confirm our assumption, we ran primary experiments resulting in no significant differences in CFUs between biofilms after 7-min treatment with $16 \%$ alcohol and the untreated control (Supplementary Fig. S1). Hence, a bactericidal effect of alcohol at the tested concentration can be excluded. It is recommended that future studies examine the role of alcohol in detail using live dead staining along with CFU determination. A bactericidal effect of the alcohol on the mature oral biofilm is not possible after an incubation time of seven minutes as detected by the CFU ${ }^{64}$. The authors reported that an alcohol concentration of $30 \%$ and incubation time of 8 hours were required to detect bactericidal effects against biofilms formed by Klebsiella pneumoniae and Pseudomonas aeruginosa after 40 hours. It should be taken into consideration that mature multispecies oral biofilms are more resistant to alcohol than mono-species biofilms tested in the aforementioned study. Ultrasonication (70\% amplitude) using a digital ultrasonic bath (Sonorex Digital $10 \mathrm{P}$ from Bandelin, Berlin, Germany) was applied to dissolve adherent bacteria from substratum. This ultrasonication power treatment for $4 \mathrm{~min}$ on ice was highly effective in killing adherent anaerobic bacteria such as Porphyromonas gingivalis, as reported in a previous own study ${ }^{65}$.

In a recent study, a promoting effect for periodontal healing was found for photosensitizer methylene blue (MB), which was dissolved in $20 \%$ ethanol during aPDT ${ }^{13,47}$. Presumably, this can be attributed to the up to 5 -fold increase in survival time of $\operatorname{ROS}^{66}$. Another study showed that $20-30 \%$ ethanol is necessary to prevent the resuscitation of multispecies biofilms ${ }^{67}$. This supportive antimicrobial effect of ethanol can be attributed to the manipulation of the bacterial membrane and the denaturation of proteins ${ }^{68}$. In earlier reports, it has been shown that lipophilic photosensitizers are much more efficient than hydrophilic ones with the same ROS supply ${ }^{69,70}$. In contrast to conventional photosensitizing agents such as TB, ICG, MB and Ce6, the high anti-bacterial efficiency of $H$. perforatum extract cannot be attributed to mechanisms involving only one specific pure substance. Interestingly, synergetic interactions between three main constituents - namely hypericin, pseudohyperin and hyperforin - are responsible for the high antimicrobial potential of $H$. perforatum ${ }^{26,27,71,72}$. Nevertheless, their exact mechanism of action remains unknown ${ }^{26,27}$. Further studies are necessary to determine the synergistic potential of specific $H$. perforatum components.

Obtaining an in situ oral biofilm enabled us to conduct the assays under more realistic experimental conditions, as already described in previous own reports ${ }^{73,74}$. Bovine enamel slabs (BES) represent a favorable habitat for the adhesion of oral microorganisms which is physicochemically similar to human tooth surfaces ${ }^{75}$. Due to substantial differences in biofilm composition and architecture between artificial and natural tooth surfaces, the tests were conducted solely on the latter.

About 700 oral bacterial species are estimated as residing within the oral cavity, of which only $50 \%$ have been cultivated thus $\mathrm{far}^{76,77}$. Subsequently, the plate count technique as well as the culture media favor the growth of certain types of bacteria ${ }^{78}$. Nevertheless, CFU remains the most widely used gold standard method for the quantification of viable bacteria ${ }^{79}$, which is the focus of this study.

The application of aPDT completely inhibited bacterial growth when the initial and three-day-old biofilms were photodynamically treated in the presence of $H$. perforatum.

This is a very interesting finding, indicating the high bactericidal potential of $H$. perforatum-mediated aPDT compared with aPDT employing other photosensitizers in previous reports ${ }^{17,76,77,80}$. The presence of extracellular 
polymeric substances (EPS) within the oral biofilm and the chemical composition of the photosensitizers and cell envelope in Gram-positive and Gram-negative bacteria are crucial parameters that can define the degree of photosensitizer diffusion in biofilm biomass. In order to investigate the exact depth of penetration as well as whether non-viable bacteria can survive this procedure, further studies are necessary. Another critical aspect is the possibility that aPDT favors the survival of resistant bacteria. Although there are no relevant studies in the literature, even if this assumption was true, the application of low concentrations of photosensitizer would carry the same risk of creating a resistance-prone microbial community as treatment with low doses of CHX or antibiotics ${ }^{1,2,81,82}$.

In this study, we have shown that treatment with CHX as well as aPDT after rinsing off $H$. perforatum reduced the number of viable bacterial species, albeit not significantly. Nevertheless, the shift to a predominately aerobic environment after aPDT holds strong importance as it implies the presence of a high alteration potential of $H$. perforatum-mediated aPDT on biofilm synthesis. Caries and periodontitis are biofilm-associated oral infections that can be controlled after favorably changing the composition of the pathogenic microbial ecosystems into healthier microbial communities ${ }^{23,77,83,84}$. Ascertaining which alterations of the biofilm composition are advantageous for the development of a 'healthy' microbial environment constitutes an alternative treatment approach in case the complete mechanical removal of the biofilm poses clinical challenges and should be the focus of future investigations $^{85-87}$.

Interestingly, $H$. perforatum had the highest efficiency as a photosensitizer when it was not rinsed prior to aPDT. This could be explained by the fact that the prolonged contact of $H$. perforatum with the oral biofilm could facilitate the maximum penetration of the biofilm through diffusion or endocytosis ${ }^{4}$. Taking the short lifetime of the ROS (about 10-320 ns) into consideration, the time extension of the synergistic interaction between diverse components in $H$. perforatum total extract and biofilm constituents such as EPS may be crucial for the aPDT-induced antimicrobial efficacy ${ }^{17,21}$. The highest antimicrobial effect can be achieved when the photosensitizer remains as close as possible to the targeted bacteria ${ }^{43,88}$.

To sum up, this study has shown that $H$. perforatum as a PS in combination with VIS and wIRA has a strong ability to eradicate in situ oral biofilms. The null hypothesis was rejected. The results of the study highlight the already-known positive effects of aPDT in the dental field and introduce $H$. perforatum as a potent photosensitizer for clinical use.

\section{Material and Methods}

Selection of study volunteers and test samples. After the review and approval of the study protocol by the Ethics Committee of the University of Freiburg (Nr. 91/13), six healthy volunteers between the age of 24 and 44 were selected and gave their written informed consent to participate in this study. All experiments and data collection were performed in accordance with relevant institutional and national guidelines and regulations. Individual clinical oral examinations were performed for each volunteer prior to study enrollment. Thus, the salivary flow rate was estimated in the range of $1.2 \pm 0.4 \mathrm{ml} / \mathrm{min}$, and the lactate production rate was measured at $2.7 \pm 0.6$ (scale from 1 to 9). The mean values for decayed, missing, filled teeth (DMFT) ranged between 0 and $7^{17,89}$. A complete dentition allowing for sufficient room for the slabs served as a requirement for the stable maintenance of the splint. The exclusion criteria included severe systematic disease, diseases of the salivary glands, carious lesions or periodontal disease, pregnancy or lactation, and use of antibiotics or local antimicrobial mouth rinses e.g. chlorhexidine ( $\mathrm{CHX}$ ) within the last 30 days ${ }^{17,80}$.

In order to perform the experiment, BES were obtained from freshly slaughtered two-year-old cattle (diameter, $5 \mathrm{~mm}$; surface area, $19.63 \mathrm{~mm}^{2}$; height, $1 \mathrm{~mm}$ ). Before extracting their teeth, the cattle were examined for the absence of bovine spongiform encephalopathy (BSE) using an IDEXX labor index diagnostic kit (Ludwigsburg, Germany). The BES were fixed into plastic holders yielding parallel surfaces with an average height of $1 \mathrm{~mm}$ and were then polished using wet sandpaper with increasing corn sizes from 250 to 40,000 grits in a special polishing machine (Knuth-Rothor-3; Streuers, Willich, Germany) to yield a smooth surface. To guarantee consistency and thereby confirm the presence of intact enamel with clean polished surfaces a light microscope (Wild M3Z, Leica $\mathrm{GmbH}$, Wetzlar, Germany) was used, and the BES were finally disinfected as previously described ${ }^{80}$. In brief, the first disinfection step was ultrasonication of the BES for 3 minutes with $\mathrm{NaOCl}$ (3\%), followed by air drying to remove the superficial smear layer. For additional disinfection, $70 \%$ ethanol was subsequently applied for three minutes under ultrasonication. In the second disinfection step, BES were fixed to acrylic oral appliances described elsewhere and both BES and the devices were ultrasonicated twice in double-distilled water for ten minutes to allow the removal of residual $\mathrm{NaOCl}$. Before the experiments, the BES-loaded oral devices were stored in distilled water for at least 24 hours to hydrate ${ }^{17}$.

Individual upper jaw acrylic devices were constructed with a total of six round openings at the interdental area between the upper premolars and molars, three on each side, respectively. The openings were sufficiently wide and deep to allow for the insertion of BES without interaction with the mucosa (Fig. 1). In order to fix the BES into the openings, an A silicon compound (Panasil initial contact X-Light, Kettenbach GmbH \& Co. KG, Eschenburg, Germany) was used. In order to ensure that only BES surfaces were exposed to the oral cavity and that no unwanted contact took place between the buccal mucosa or the tongue and the BES, the BES margins were fully covered with silicon. In order to yield a sufficient number of biofilm samples for the aPDT assays, each volunteer carried the appliances loaded with a total of twelve BES twice, for two hours and three days, respectively.

Radiation source and photosensitizer. The obtained biofilm samples were illuminated by broadband VIS + wIRA radiator (Hydrosun 750 FS, Hydrosun Medizintechnik GmbH, Müllheim, Germany) containing a $7 \mathrm{~mm}$ water cuvette ${ }^{14,17}$. For the absorption of the infrared-B and $-\mathrm{C}$ wavelengths emitted by the halogen lamp, an accessory water filter (dimensions: length: $28 \mathrm{~cm}$, width: $27 \mathrm{~cm}$, height: $28 \mathrm{~cm}$ ) using $750 \mathrm{~W}$ (voltage: $230 \mathrm{~V}, 50-60 \mathrm{~Hz}$ ) was inserted into the light path. The radiator also included an orange filter, namely BTE 31 , with a diameter of $10 \mathrm{~cm}$ and an efficiency level of $200 \mathrm{~mW} / \mathrm{cm}^{2}$. The BTE 31 filter enabled more than a 
doubled-weighted efficient integral illumination with reference to the absorption spectrum of protoporphyrin IX. In order to prevent superficial overheating, the absorption bands 944 and $1180 \mathrm{~nm}$ were also filtered. The total spectrum of the water-filtered absorption ranged from $570 \mathrm{~nm}$ to $1400 \mathrm{~nm}$, with local minima at around $970 \mathrm{~nm}$, $1200 \mathrm{~nm}$ and $1430 \mathrm{~nm}$, respectively ${ }^{90}$. The total amount of unweighted (absolute) radiation applied to the biofilms for 5 minutes equaled $200 \mathrm{~mW} \mathrm{~cm}^{-2}$ VIS + wIRA, which comprised $48 \mathrm{~mW} \mathrm{~cm}^{-2}$ VIS and $152 \mathrm{~mW} \mathrm{~cm}^{-2}$ wIRA. This broad-spectrum light source provided optimal absorption for the tested photosensitizer, namely $H$. perforatum ethanol (15.8\%) extract (St. John's Wort, Quality Herbal Extracts, Cooranbong, Australia). Pseudohypericin - the main component of $H$. perforatum - can be activated within the UVA (and UVA1) visible absorption range, while absorption peaks are mainly exhibited at red wavelengths $(570-700 \mathrm{~nm})^{91}$. The visible absorption maximum $\left(\lambda_{\max }\right)$ of $H$. perforatum is $598 \mathrm{~nm}^{92}$. For the assays, $H$. perforatum extract was diluted in $0.9 \%$ saline $(\mathrm{NaCl})$ to a final concentration of $32 \mathrm{mg} \mathrm{ml}^{-1}$ and a final alcohol concentration of $15.8 \%(\mathrm{v}$ : v). Prior to use, the original extract solution was stored in a light safety glass at $4{ }^{\circ} \mathrm{C}$ in the dark for up to fourteen days to block any light-induced photochemical attenuation.

Extraction of $\boldsymbol{H}$. perforatum. Wild-growing Hypericum perforatum was harvested during the flowering phase. The herbaceous material was dried, cut, and stored until further processing. The crude extract was produced by percolation using $95 \%$ aqueous ethanol as a solvent (menstruum). The milled herbal material was moistened with some of the menstruum and packed firmly in a cylindrical percolator. Additional menstruum was added to the percolator and the herb was allowed to macerate for 24 hours before the percolation was commenced. Percolation comprised the slow passage of menstruum through the column of drug. The maceration and percolation were performed at $30^{\circ} \mathrm{C}$. The percolation was repeated until the material was exhausted. The extractions were combined and the solvent recovered under vacuum evaporation to yield a final ratio of one part drug per one part solvent $(1: 1)$.

Ultra-high-performance liquid chromatography coupled with high resolution mass spectrometry (UPLC-HRMS). The H. perforatum extract was submitted to UPLC-HRMS/MS analysis on an AQUITY system (Waters) connected to an LTQ-OrbitrapR XL hybrid mass spectrometer (Thermo Scientific) equipped with an electrospray ionization (ESI) source and operated in negative mode. A UPLC separation gradient was developed to efficiently resolve all compounds for a qualitative analysis. The flow rate was set at $0.4 \mathrm{~mL} / \mathrm{min}$ and the solvent system was (A) water $0.1 \%$ formic acid and (B) acetonitrile $0.1 \%$ formic acid. The elution program was $2 \%$ B for 2 minutes, $100 \%$ B for 18 minutes, and held for 2 minutes. After returning to $2 \%$ B for 1 minute, column equilibration was performed for 4 minutes at the end of the run. The injection volume was set to $10 \mu \mathrm{L}$ and samples were injected at $0.2 \mathrm{mg} / \mathrm{mL}$ in water-acetonitrile solution $(1: 1)$ on a Fortis, $\mathrm{C} 18(100 \times 2.1 \mathrm{~mm}$ i.d, 1.7 $\mu \mathrm{m}$ particle size). The HRMS and HRMS/MS data were acquired in negative mode over a $100-1000 \mathrm{~m} / \mathrm{z}$ range. The MS profile was recorded in full-scan mode ( The ESI conditions were as follows: capillary temperature $320^{\circ} \mathrm{C}$; capillary voltage $-40 \mathrm{~V}$; tube lens $-120 \mathrm{~V}$; ESI voltage $2.7 \mathrm{kV}$. Nitrogen was used as sheath gas (40 Au) and auxiliary gas (8 Au). For the HRMS/MS acquisitions, a data-dependent method including the detection (full scan) and fragmentation of the three most intense peaks per scan was used. The mass resolving power was 30,000 for both levels and the normalized collision energy (CID) in the ion trap was set to $35.0 \%(\mathrm{q}=0.25)$ for the HRMS/MS experiments. Chromatographic and spectrometric features were used for identifying extracts constituents such as retention time (Rt), polarity, accurate $\mathrm{m} / \mathrm{z}$, proposed elemental composition (EC) and ring double bond equivalent (RDBeq) values as well as HRMS/ MS spectra and derived fragmentation motifs. The raw data were acquired and processed using XCalibur 2.2.4 software from Thermo Scientific.

aPDT protocol for in situ oral biofilms. Each participant twice carried an individual BES-loaded upper jaw acrylic device for the assays involving the initial adhesion (two hours) and biofilm formation (three days). After obtaining the in situ biofilms, the devices were removed from the oral cavity. Subsequently, BES were detached using sterile tweezers and a dental probe to avoid disturbing the intact in situ biofilms. Each of the BES was rinsed for 30 seconds with sterile $0.9 \% \mathrm{NaCl}$ to ensure the displacement of non-adherent bacteria. One BES served as a positive control and was treated with $0.2 \% \mathrm{CHX}$, while another two BES treated with $0.9 \% \mathrm{NaCl}$ and $H$. perforatum without aPDT were used as negative controls. One biofilm-covered BES was preincubated in $H$. perforatum, which was rinsed off for 30 seconds with $1 \mathrm{ml} 0.9 \% \mathrm{NaCl}$ prior to aPDT ex vivo using VIS + wIRA to allow for optimal depth of the irradiation. The remaining BES was also preincubated in $H$. perforatum, which remained during aPDT ex vivo using VIS + wIRA. The preincubation time for each BES in H. perforatum at a concentration of $32 \mathrm{mg} \mathrm{ml}^{-1}$ was two minutes in multiwell plates (24-well plate, Greiner bio-one $\mathrm{GmbH}$, Frickenhausen, Germany) in the dark as described elsewhere ${ }^{17}$, while the irradiation by VIS + wIRA lasted five minutes at $37^{\circ} \mathrm{C}$ (Fig. 2). After aPDT, the BES were inserted into multiwell plates with $1 \mathrm{ml} 0.9 \% \mathrm{NaCl}$ and the quantification and identification of adherent microorganisms were performed by the CFU and with MALDI-TOF, respectively. The activity of the photosensitizer remained stable for six months of storage at low temperature $\left(3^{\circ} \mathrm{C}\right)$ in the dark using a light-tight bottle. The participants carried the BES twice for $72 \mathrm{~h}$ and $2 \mathrm{~h}$, respectively.

Quantification of the adherent oral microorganisms within biofilms. The margins and the bottom dentine surfaces of the biofilm-loaded BES were brushed down with small sterile foam pellets (Voco GmbH, Cuxhaven, Germany) to displace non-adherent microorganisms. Subsequently, the BES were washed with $0.9 \%$ $\mathrm{NaCl}$ for at least 10 seconds to remove the remaining non-attached microorganisms. For the dilution series, each treated BES was transferred into Eppendorf tubes (Eppendorf $\mathrm{GmbH}$, Wesseling-Berzdorf, Germany) containing $1 \mathrm{ml} 0.9 \% \mathrm{NaCl}$, ultrasonicated for 3 minutes in $1 \mathrm{ml} \mathrm{NaCl}$ on ice and finally vortexed for at least 30 seconds to dislodge the biofilm microorganisms from the BES surface. Subsequently, the suspensions untreated BES (negative 
control), CHX-treated BES (positive control) and all aPDT-treated BES were diluted from up to 1:10 ${ }^{3}$ (initial biofilm) and up to $1: 10^{6}$ (mature biofilm) in $0.9 \% \mathrm{NaCl}$. From each dilution series of the differently treated BES three different dilutions were plated on separate agar plates. Thereafter, the microorganisms were cultivated and identified as previously described ${ }^{93}$. In brief, for the CFU estimation of the aerobic and facultative anaerobic bacteria Columbia blood agar plates (CBA; Becton, Dickinson, Heidelberg, Germany) were used at $37^{\circ} \mathrm{C}$ and $5 \% \mathrm{CO}_{2}$ for 3-5 days. Anaerobic bacteria were cultivated on yeast-cystein blood agar plates (HCB; Becton, Dickinson, Heidelberg, Germany) and incubated for ten days at $37^{\circ} \mathrm{C}$ (anaerobic chamber; Genbox bioMérieux SA, Marcy $l^{\prime}$ Etoile, France). The CFU were finally counted per $\mathrm{ml}$ of the original suspension using Gel Doc EQ universal hood (Bio-Rad Life Science Group, Hercules, CA, USA). Each measurement was repeated, and the surviving bacteria were counted by eye if necessary.

Identification of the adherent oral microorganisms within biofilms. The recovered bacterial colonies were sub-cultivated to yield pure cultures. In order to identify certain bacterial species, MALDI-TOF analysis in a MALDI Biotyper Microflex LT (Maldi Biotyper, Bruker Daltonik GmbH, Bremen, Germany) was applied. The Biotyper 3.0 Software obtained mass spectra according to the manufacturer's instructions and compared the acquired spectra with a total of 3,740 spectra of the reference database (representing 319 genera and 1,946 species). The resulting similarities were illustrated by a log score indicating the species level with values $\geq 2.0$, whereas values $\geq 1.7$ represented identification at the genus level. For values $<1.7$, the yielded spectrum exhibited no significant similarity to any database spectrum. The analysis was repeated if the identification of the species could not be confirmed. Moreover, universal bacterial PCR was performed as a supplementary method to identify the surviving oral microorganisms. In the presence of MALDI-TOF scores $<1.7$, the following primers were applied: TP16U1: 5'-AGAGTTTGATCMTGGCTCAG-3' and RT16U6: 5'-ATTGTAGCACGTGTGTNCCCC-3' followed by sequencing on a 3130 Genetic Analyzer (Applied Biosystems, Life Technologies GmbH, Darmstadt, Germany).

Statistical analysis. The effects of aPDT using VIS + wIRA and H. perforatum as a photosensitizer were calculated in a detailed evaluation for all six volunteers. For descriptive analysis, mean and standard deviations were computed. For graphical demonstration of the results, boxplots were used. Diagrams that demonstrated the $\log _{10}$ scale per square centimeter $\left(\log _{10} / \mathrm{cm}^{2}\right)$ were selected to visualize the viable bacteria stratified to biofilm age (initial/mature) and type of microorganism (aerobic/anaerobic). A Friedman test was used to depict the differences between the aPDT-treated groups and the controls. Because a nonparametric test lacks sufficient power due to the small number of cases, paired $t$-tests with Bonferroni correction (multiple testing) were used for pairwise tests of differences among biofilms. Scheffés method was applied to correct the multiple testing errors through the adjustment of p-values. The statistical analysis was performed using STATA 14.

\section{Data availability}

All data generated or analyzed during this study are included in this published article.

Received: 5 August 2019; Accepted: 19 December 2019;

Published online: 30 December 2019

\section{References}

1. Hol, F. J. H., Hubert, B., Dekker, C. \& Keymer, J. E. Density-dependent adaptive resistance allows swimming bacteria to colonize an antibiotic gradient. ISME J 10, 30-38 (2016).

2. Murray, A. K. et al. Novel Insights into Selection for Antibiotic Resistance in Complex Microbial Communities. MBio 9, (2018).

3. Sukumar, S., Roberts, A. P., Martin, F. E. \& Adler, C. J. Metagenomic Insights into Transferable Antibiotic Resistance in Oral Bacteria. J. Dent. Res. 95, 969-976 (2016).

4. Chambless, J. D., Hunt, S. M. \& Stewart, P. S. A Three-Dimensional Computer Model of Four Hypothetical Mechanisms Protecting Biofilms from Antimicrobials. Appl Environ Microbiol 72, 2005-2013 (2006).

5. Takasaki, A. A. et al. Application of antimicrobial photodynamic therapy in periodontal and peri-implant diseases. Periodontol. 2000 51, 109-140 (2009).

6. Cieplik, F. et al. Photodynamic biofilm inactivation by SAPYR-an exclusive singlet oxygen photosensitizer. Free Radic. Biol. Med. 65, $477-487$ (2013).

7. Maisch, T., Szeimies, R.-M., Jori, G. \& Abels, C. Antibacterial photodynamic therapy in dermatology. Photochem. Photobiol. Sci. 3, 907-917 (2004).

8. Beltes, C., Sakkas, H., Economides, N. \& Papadopoulou, C. Antimicrobial photodynamic therapy using Indocyanine green and near-infrared diode laser in reducing Entrerococcus faecalis. Photodiagnosis Photodyn Ther 17, 5-8 (2017).

9. Hartel, M. et al. Randomized clinical trial of the influence of local water-filtered infrared A irradiation on wound healing after abdominal surgery. Br J Surg 93, 952-960 (2006).

10. Künzli, B. M. et al. Impact of preoperative local water-filtered infrared A irradiation on postoperative wound healing: a randomized patient- and observer-blinded controlled clinical trial. Ann. Surg. 258, 887-894 (2013).

11. Jung, T. \& Grune, T. Experimental basis for discriminating between thermal and athermal effects of water-filtered infrared A irradiation. Ann. N. Y. Acad. Sci. 1259, 33-38 (2012).

12. Cieplik, F., Tabenski, L., Buchalla, W. \& Maisch, T. Antimicrobial photodynamic therapy for inactivation of biofilms formed by oral key pathogens. Front Microbiol 5, (2014).

13. Prochnow, E. P. et al. Antimicrobial photodynamic effect of phenothiazinic photosensitizers in formulations with ethanol on Pseudomonas aeruginosa biofilms. Photodiagnosis Photodyn Ther 13, 291-296 (2016).

14. Al-Ahmad, A. et al. Antimicrobial photodynamic therapy using visible light plus water-filtered infrared-A (wIRA). J. Med. Microbiol. 62, 467-473 (2013).

15. Al-Ahmad, A. et al. Photoinactivation Using Visible Light Plus Water-Filtered Infrared-A (vis+wIRA) and Chlorine e6 (Ce6) Eradicates Planktonic Periodontal Pathogens and Subgingival Biofilms. Front Microbiol 7, 1900 (2016).

16. Karygianni, L. et al. Natural Antimicrobials and Oral Microorganisms: A Systematic Review on Herbal Interventions for the Eradication of Multispecies Oral Biofilms. Front Microbiol 6, 1529 (2015). 
17. Karygianni, L. et al. Novel Broad-Spectrum Antimicrobial Photoinactivation of In Situ Oral Biofilms by Visible Light plus WaterFiltered Infrared A. Appl. Environ. Microbiol. 80, 7324-7336 (2014).

18. Wölfle, U., Seelinger, G. \& Schempp, C. M. Topical application of St. John’s wort (Hypericum perforatum). Planta Med. 80, 109-120 (2014).

19. Briskin, D. P. Medicinal plants and phytomedicines. Linking plant biochemistry and physiology to human health. Plant Physiol. 124, 507-514 (2000)

20. Karioti, A. \& Bilia, A. R. Hypericins as potential leads for new therapeutics. Int J Mol Sci 11, 562-594 (2010).

21. Gaedcke, F. [The example: St. John's wort. Manufacturing and quality aspects of plant extracts]. Pharm Unserer Zeit 32, 192-201 (2003).

22. Theodossiou, T. A., Hothersall, J. S., De Witte, P. A., Pantos, A. \& Agostinis, P. The multifaceted photocytotoxic profile of hypericin. Mol. Pharm. 6, 1775-1789 (2009).

23. Filoche, S., Wong, L. \& Sissons, C. H. Oral biofilms: emerging concepts in microbial ecology. J. Dent. Res. 89, 8-18 (2010).

24. Carpenter, D. J. St. John's wort and S-adenosyl methionine as 'natural' alternatives to conventional antidepressants in the era of the suicidality boxed warning: what is the evidence for clinically relevant benefit? Altern Med Rev 16, 17-39 (2011).

25. Amano, A. Molecular interaction of Porphyromonas gingivalis with host cells: implication for the microbial pathogenesis of periodontal disease. J. Periodontol. 74, 90-96 (2003).

26. Russo, E. et al. Hypericum perforatum: pharmacokinetic, mechanism of action, tolerability, and clinical drug-drug interactions. Phytother Res 28, 643-655 (2014).

27. Stojanović, G., Đorđević, A. \& Šmelcerović, A. Do other Hypericum species have medical potential as St. John’s wort (Hypericum perforatum)? Curr. Med. Chem. 20, 2273-2295 (2013).

28. Onoue, S. et al. In vitro photochemical and phototoxicological characterization of major constituents in St. John's Wort (Hypericum perforatum) extracts. Phytochemistry 72, 1814-1820 (2011).

29. Collins, T. L., Markus, E. A., Hassett, D. J. \& Robinson, J. B. The effect of a cationic porphyrin on Pseudomonas aeruginosa biofilms. Curr. Microbiol. 61, 411-416 (2010).

30. Chalatsa, I. et al. Beneficial Effects of Sideritis scardica and Cichorium spinosum against Amyloidogenic Pathway and Tau Misprocessing in Alzheimer's Disease Neuronal Cell Culture Models. J. Alzheimers Dis. 64, 787-800 (2018).

31. Porzel et al. - 2014 - Metabolite profiling and fingerprinting of Hyperic.pdf.

32. Sakavitsi, M.-E. et al. Comparative HPLC-DAD and UHPLC-ESI(-)-HRMS \& MS/MS profiling of Hypericum species and correlation with necrotic cell-death activity in human leukemic cells. Phytochemistry Letters 20, 481-490 (2017).

33. Hu, X., Huang, Y.-Y., Wang, Y., Wang, X. \& Hamblin, M. R. Antimicrobial Photodynamic Therapy to Control Clinically Relevant Biofilm Infections. Front Microbiol 9, 1299 (2018).

34. Nardini, E. F. et al. The potential of commercially available phytotherapeutic compounds as new photosensitizers for dental antimicrobial PDT: A photochemical and photobiological in vitro study. Photodiagnosis Photodyn Ther 27, 248-254 (2019).

35. Süntar, I., Oyardı, O., Akkol, E. K. \& Ozçelik, B. Antimicrobial effect of the extracts from Hypericum perforatum against oral bacteria and biofilm formation. Pharm Biol 54, 1065-1070 (2016).

36. Tian, J. et al. Antidepressant-like activity of adhyperforin, a novel constituent of Hypericum perforatum L. Sci Rep 4, 5632 (2014).

37. Saddiqe, Z., Naeem, I. \& Maimoona, A. A review of the antibacterial activity of Hypericum perforatum L. J Ethnopharmacol 131, 511-521 (2010).

38. Khadem Nezhad, S., Taghavi Zenouz, A., Aghazadeh, M. \& Samadi Kafil, H. Strong antimicrobial activity of Hypericum perforatum L. against oral isolates of Lactobacillus spp. Cell. Mol. Biol. (Noisy-le-grand) 63, 58-62 (2017).

39. Schumann, H., Calow, T., Weckesser, S., Müller, M. L. \& Hoffmann, G. Water-filtered infrared A for the treatment of chronic venous stasis ulcers of the lower legs at home: a randomized controlled blinded study. Br. J. Dermatol. 165, 541-551 (2011).

40. von Felbert, V. et al. Photodynamic therapy of multiple actinic keratoses: reduced pain through use of visible light plus water-filtered infrared A compared with light from light-emitting diodes. Br. J. Dermatol. 163, 607-615 (2010).

41. Konopka, K. \& Goslinski, T. Photodynamic therapy in dentistry. J. Dent. Res. 86, 694-707 (2007)

42. Agostinis, P. et al. Photodynamic therapy of cancer: an update. CA Cancer J Clin 61, 250-281 (2011).

43. Dysart, J. S. \& Patterson, M. S. Characterization of Photofrin photobleaching for singlet oxygen dose estimation during photodynamic therapy of MLL cells in vitro. Phys Med Biol 50, 2597-2616 (2005).

44. Jung, T., Höhn, A., Piazena, H. \& Grune, T. Effects of water-filtered infrared A irradiation on human fibroblasts. Free Radic. Biol. Med. 48, 153-160 (2010).

45. Giehl, K. A., Kriz, M., Grahovac, M., Ruzicka, T. \& Berking, C. A controlled trial of photodynamic therapy of actinic keratosis comparing different red light sources. Eur J Dermatol 24, 335-341 (2014).

46. Fumes, A. C., da Silva Telles, P. D., Corona, S. A. M. \& Borsatto, M. C. Effect of aPDT on Streptococcus mutans and Candida albicans present in the dental biofilm: Systematic review. Photodiagnosis Photodyn Ther 21, 363-366 (2018).

47. Pillusky, F. M. et al. Antimicrobial photodynamic therapy with photosensitizer in ethanol improves oxidative status and gingival collagen in a short-term in periodontitis. Photodiagnosis Photodyn Ther 19, 119-127 (2017).

48. Karu, T. I. Multiple roles of cytochrome c oxidase in mammalian cells under action of red and IR-A radiation. IUBMB Life 62 , 607-610 (2010)

49. Marrelli, M. et al. Hypericum perforatum: Influences of the habitat on chemical composition, photo-induced cytotoxicity, and antiradical activity. Pharm Biol 52, 909-918 (2014).

50. Kacerovská, D., Pizinger, K., Majer, F. \& Smíd, F. Photodynamic therapy of nonmelanoma skin cancer with topical hypericum perforatum extract-a pilot study. Photochem. Photobiol. 84, 779-785 (2008).

51. Kleemann, B., Loos, B., Scriba, T. J., Lang, D. \& Davids, L. M. St John's Wort (Hypericum perforatum L.) photomedicine: hypericinphotodynamic therapy induces metastatic melanoma cell death. PLoS ONE 9, e103762 (2014).

52. Menichini, G. et al. Hypericum perforatum L. subsp. perforatum induces inhibition of free radicals and enhanced phototoxicity in human melanoma cells under ultraviolet light. Cell Prolif. 46, 193-202 (2013).

53. Marrelli, M., Statti, G., Conforti, F. \& Menichini, F. New Potential Pharmaceutical Applications of Hypericum Species. Mini Rev Med Chem 16, 710-720 (2016).

54. Assiri, K., Alyami, Y., Uyanik, J. M. \& Romero-Reyes, M. Hypericum perforatum (St. John's Wort) as a possible therapeutic alternative for the management of trigeminal neuralgia (TN) - A case report. Complement Ther Med 30, 36-39 (2017).

55. Pia Schiavone, B. I. et al. Anticancer and Antibacterial Activity of Hyperforin and Its Derivatives. Anticancer Agents Med Chem 14, 1397-1401 (2014).

56. Galeotti, N. Hypericum perforatum (St John's wort) beyond depression: A therapeutic perspective for pain conditions. J Ethnopharmacol 200, 136-146 (2017).

57. Kober, M., Pohl, K. \& Efferth, T. Molecular mechanisms underlying St. John's wort drug interactions. Curr. Drug Metab. 9, 1027-1037 (2008).

58. Henderson, L., Yue, Q. Y., Bergquist, C., Gerden, B. \& Arlett, P. St John's wort (Hypericum perforatum): drug interactions and clinical outcomes. Br J Clin Pharmacol 54, 349-356 (2002).

59. Schempp, C. M., Müller, K., Winghofer, B., Schulte-Mönting, J. \& Simon, J. C. Single-dose and steady-state administration of Hypericum perforatum extract (St John's Wort) does not influence skin sensitivity to UV radiation, visible light, and solar-simulated radiation. Arch Dermatol 137, 512-513 (2001). 
60. Dikmen, M. et al. Evaluation of the wound healing potentials of two subspecies of Hypericum perforatum on cultured NIH3T3 fibroblasts. Phytother Res 25, 208-214 (2011).

61. Committee on Herbal Medicinal Products (HMPD). Assessment Report on Hypericum Perforatum L. HERBA, European Medicines Agency. Evaluation of Medicins for Human Use. Doc. Ref.: EMA/HMPC/101303/2008 p25. (2009).

62. Klein, S. D., Sandig, A., Baumgartner, S. \& Wolf, U. Differences in Median Ultraviolet Light Transmissions of Serial Homeopathic Dilutions of Copper Sulfate, Hypericum perforatum, and Sulfur. Evid Based Complement Alternat Med 2013, 370609 (2013).

63. Liu, F. F., Ang, C. Y. \& Springer, D. Optimization of extraction conditions for active components in Hypericum perforatum using response surface methodology. J. Agric. Food Chem. 48, 3364-3371 (2000).

64. Chambers, S. T., Peddie, B. \& Pithie, A. Ethanol disinfection of plastic-adherent micro-organisms. J. Hosp. Infect. 63, 193-196 (2006).

65. Al-Ahmad, A. et al. Bacterial and Candida albicans adhesion on rapid prototyping-produced 3D-scaffolds manufactured as bone replacement materials. J Biomed Mater Res A 87, 933-943 (2008).

66. Meisel, P. \& Kocher, T. Photodynamic therapy for periodontal diseases: State of the art. Journal of Photochemistry and Photobiology B: Biology 79, 159-170 (2005).

67. Peters, B. M., Ward, R. M., Rane, H. S., Lee, S. A. \& Noverr, M. C. Efficacy of Ethanol against Candida albicans and Staphylococcus aureus Polymicrobial Biofilms. Antimicrobial Agents and Chemotherapy 57, 74-82 (2013).

68. McDonnell, G. \& Russell, A. D. Antiseptics and Disinfectants: Activity, Action, and Resistance. Clinical Microbiology Reviews 12, 147-179 (1999).

69. Moan, J. et al. Photosensitizing efficiencies, tumor- and cellular uptake of different photosensitizing drugs relevant for photodynamic therapy of cancer. Photochem. Photobiol. 46, 713-721 (1987).

70. Peng, Q. et al. Subcellular localization, redistribution and photobleaching of sulfonated aluminum phthalocyanines in a human melanoma cell line. Int. J. Cancer 49, 290-295 (1991)

71. Lawvere, S. \& Mahoney, M. C. St. John’s wort. Am Fam Physician 72, 2249-2254 (2005).

72. Zhao, J., Liu, W. \& Wang, J.-C. Recent advances regarding constituents and bioactivities of plants from the genus Hypericum. Chem. Biodivers. 12, 309-349 (2015).

73. Al-Ahmad, A. et al. In vivo study of the initial bacterial adhesion on different implant materials. Arch. Oral Biol. 58, 1139-1147 (2013).

74. Al-Ahmad, A. et al. Bacterial colonization of enamel in situ investigated using fluorescence in situ hybridization. J. Med. Microbiol. 58, 1359-1366 (2009).

75. Teles, F. R. et al. Comparison of microbial changes in early redeveloping biofilms on natural teeth and dentures. J. Periodontol. 83, 1139-1148 (2012).

76. Aas, J. A., Paster, B. J., Stokes, L. N., Olsen, I. \& Dewhirst, F. E. Defining the normal bacterial flora of the oral cavity. J. Clin. Microbiol. 43, 5721-5732 (2005)

77. Kumar, P. S. et al. Changes in periodontal health status are associated with bacterial community shifts as assessed by quantitative $16 \mathrm{~S}$ cloning and sequencing. J. Clin. Microbiol. 44, 3665-3673 (2006).

78. Amann, R. I., Ludwig, W. \& Schleifer, K. H. Phylogenetic identification and in situ detection of individual microbial cells without cultivation. Microbiol. Rev. 59, 143-169 (1995).

79. Hazan, R., Que, Y.-A., Maura, D. \& Rahme, L. G. A method for high throughput determination of viable bacteria cell counts in 96well plates. BMC Microbiol. 12, 259 (2012).

80. Al-Ahmad, A. et al. Antimicrobial Photoinactivation Using Visible Light Plus Water-Filtered Infrared-A (VIS + wIRA) Alters In Situ Oral Biofilms. PLoS ONE 10, e0132107 (2015)

81. Proksch, S. et al. Melatonin as a candidate therapeutic drug for protecting bone cells from chlorhexidine-induced damage. J. Periodontol. 85, e379-389 (2014).

82. Kitagawa, H. et al. Evolution of resistance to cationic biocides in Streptococcus mutans and Enterococcus faecalis. J Dent 47, 18-22 (2016).

83. Beighton, D. Can the ecology of the dental biofilm be beneficially altered? Adv. Dent. Res. 21, 69-73 (2009)

84. Liu, B. et al. Deep sequencing of the oral microbiome reveals signatures of periodontal disease. PLoS ONE 7, e37919 (2012).

85. Haffajee, A. D., Socransky, S. S., Patel, M. R. \& Song, X. Microbial complexes in supragingival plaque. Oral Microbiol. Immunol. 23, 196-205 (2008).

86. Socransky, S. S., Haffajee, A. D., Cugini, M. A., Smith, C. \& Kent, R. L. Microbial complexes in subgingival plaque. J. Clin. Periodontol. 25, 134-144 (1998).

87. Bowen, W. H. \& Koo, H. Biology of Streptococcus mutans-derived glucosyltransferases: role in extracellular matrix formation of cariogenic biofilms. Caries Res. 45, 69-86 (2011).

88. Moan, J. et al. Intracellular localization of photosensitizers. Ciba Found. Symp. 146, 95-107; discussion 107-111 (1989).

89. Kharkwal, G. B., Sharma, S. K., Huang, Y.-Y., Dai, T. \& Hamblin, M. R. Photodynamic therapy for infections: clinical applications. Lasers Surg Med 43, 755-767 (2011).

90. Piazena, H. \& Kelleher, D. K. Effects of infrared-A irradiation on skin: discrepancies in published data highlight the need for an exact consideration of physical and photobiological laws and appropriate experimental settings. Photochem. Photobiol. 86, 687-705 (2010).

91. Beattie, P. E. et al. Can St John's wort (hypericin) ingestion enhance the erythemal response during high-dose ultraviolet A1 therapy? Br. J. Dermatol. 153, 1187-1191 (2005).

92. Uzdensky, A. B. et al. Intracellular localisation of hypericin in human glioblastoma and carcinoma cell lines. Lasers Med Sci 16, 276-283 (2001)

93. Anderson, A. C. et al. Comprehensive analysis of secondary dental root canal infections: a combination of culture and cultureindependent approaches reveals new insights. PLoS ONE 7, e49576 (2012).

\section{Acknowledgements}

This study was supported by the Swiss Dr. Braun Science Foundation and partly by the German Research Foundation (DFG, AL-1179/2-1). Bettina Spitzmüller is acknowledged for her excellent technical assistance.

\section{Author contributions}

A.V. conducted the experiments and wrote the manuscript, A.A.A. participated in the conception of the study and data analysis, A.A., A.W., and K.F. conducted the experiments and were involved in data analysis and manuscript drafting, T.T., E.H., T.A., and A.L.S. were actively involved in data analysis and critically reviewed the manuscript, K.V. performed the statistical analysis and reviewed the manuscript, L.K. participated in the design of the study and data interpretation and critically reviewed the manuscript. All authors have read and approved the manuscript.

\section{Competing interests}

The authors declare no competing interests. 


\section{Additional information}

Supplementary information is available for this paper at https://doi.org/10.1038/s41598-019-56925-7.

Correspondence and requests for materials should be addressed to L.K.

Reprints and permissions information is available at www.nature.com/reprints.

Publisher's note Springer Nature remains neutral with regard to jurisdictional claims in published maps and institutional affiliations.

(c) (i) Open Access This article is licensed under a Creative Commons Attribution 4.0 International License, which permits use, sharing, adaptation, distribution and reproduction in any medium or format, as long as you give appropriate credit to the original author(s) and the source, provide a link to the Creative Commons license, and indicate if changes were made. The images or other third party material in this article are included in the article's Creative Commons license, unless indicated otherwise in a credit line to the material. If material is not included in the article's Creative Commons license and your intended use is not permitted by statutory regulation or exceeds the permitted use, you will need to obtain permission directly from the copyright holder. To view a copy of this license, visit http://creativecommons.org/licenses/by/4.0/.

(c) The Author(s) 2019 